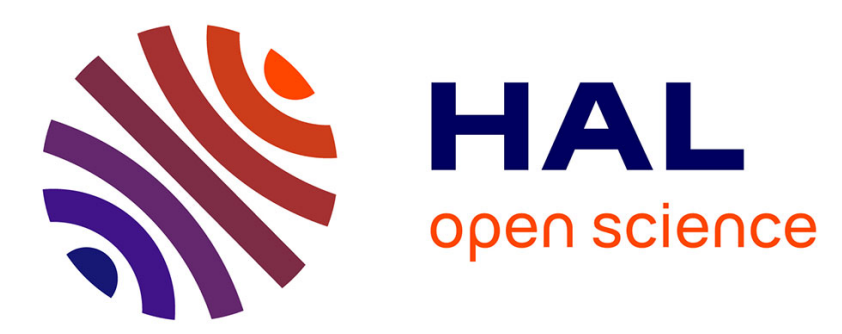

\title{
Jump in the conduction heat flux at the gas/solid interface in micro-channels
}

Dahia Chibouti, Benoît Trouette, Eric Chénier

\section{To cite this version:}

Dahia Chibouti, Benoît Trouette, Eric Chénier. Jump in the conduction heat flux at the gas/solid interface in micro-channels. International Journal of Thermal Sciences, 2021, 159. hal-02930894

\section{HAL Id: hal-02930894 \\ https://hal.science/hal-02930894}

Submitted on 4 Sep 2020

HAL is a multi-disciplinary open access archive for the deposit and dissemination of scientific research documents, whether they are published or not. The documents may come from teaching and research institutions in France or abroad, or from public or private research centers.
L'archive ouverte pluridisciplinaire HAL, est destinée au dépôt et à la diffusion de documents scientifiques de niveau recherche, publiés ou non, émanant des établissements d'enseignement et de recherche français ou étrangers, des laboratoires publics ou privés. 


\title{
Jump in the conduction heat flux at the gas/solid interface in micro-channels
}

\author{
Dahia Chibouti $\bowtie$, Benoît Trouette, Eric Chénier \\ \{dahia.chibouti; benoit.trouette; eric.chenier\}@univ-eiffel.fr \\ MSME, Univ Gustave Eiffel, CNRS UMR 8208, \\ Univ Paris Est Creteil, F-77474 Marne-la-Vallée, France \\ Version: September 4, 2020
}

\section{Contents}

1 Introduction 1

2 Continuum description $\quad 2$

3 Microscopic approach 2

4 Results and discussions $\mathbf{3}$

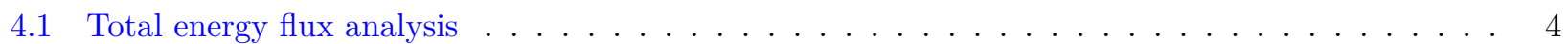

4.2 Comparison between the molecular and continuum models . . . . . . . . . . . . . . . . 5

5 Conclusion

\begin{abstract}
In micro-channel gas flows, velocity slip and temperature jump must be applied at the fluid/solid interface to take the thermodynamic non-equilibrium into account in the vicinity of the wall. In this work, simulations at molecular scale have been carried out and shown a jump in the conduction heat flux, in agreement with the boundary condition proposed by Maslen [10] in continuum mechanics.
\end{abstract}

Keywords: Heat transfer; Micro-channel; Microfluidics; Rarefied gas; Slip flow; Temperature jump; Heat flux jump

\section{Introduction}

The understanding of fluid flows and heat transfer at scales of few microns is of paramount importance since the development of Micro-electro-mechanical Systems (MEMS). When the characteristic length scale of the flow decreases to reach about 10 times the free mean path $\lambda$ of the gas molecules, the usual NavierStokes and energy equations remain valid in the core flow, but careful attention must be paid on the gas/solid boundary conditions. In a fluid layer of order $\lambda$ close to the wall, the so-called Knudsen layer, the gas molecules are no more in a local thermodynamic equilibrium: the macroscopic model based on the continuity of the velocity, temperature and heat flux at the interface between the gas and the wall falls and slip or jump conditions have to be used, like those independently proposed by Navier [12] and later on by Maxwell [11]. Whereas the boundary conditions applied on the velocity and temperature are quite well es- tablished in the 'gaseous microfluidic' community, that associated to the jump in the conduction heat flux at the wall due to the viscous friction, and first proposed by Maslen [10] in 1958, has often been neglected, forgotten or misunderstood, including in late papers. In the recent works by X. Nicolas et al. [15], a review of few papers using or ignoring the Maslen's flux condition is performed, followed by its proof which relies on the energy flux conservation principle at the macroscale. The same authors also showed that the use of the Maslen's flux condition explains the very small Nusselt number values in experiments performed on microfluidic gaseous flows [14]. However, their mathematical approach is questionable since it relies on the assumption of the continuum mechanics which is valid far from the boundaries but seems no more applicable in the Knudsen layer.

This short contribution aims to check if the Maslen's flux condition at the gas/solid interface is correct by getting rid of the continuum model and by simulat- 
ing the fluid flow and energy transfer at the molecular scale.

\section{Continuum description}

An established argon (Ar) fluid flow between two infinite $(x, y)$-parallel solid walls of platinum $(\mathrm{Pt})$ is considered (Fig. 1). Walls are distant from $H$ and their thickness is $e$. The flow is created by a volume force $\mathbf{g}=g_{x} \mathbf{e}_{x}$ acting as a uniform pressure gradient in $x$ direction. The platinum wall starting at $z=H / 2$ is thermally isolated, i.e. the conduction heat flux $q_{z}=0$, whereas the one ending at $z=-H / 2$ is isotherm, at the temperature $T_{1}$.

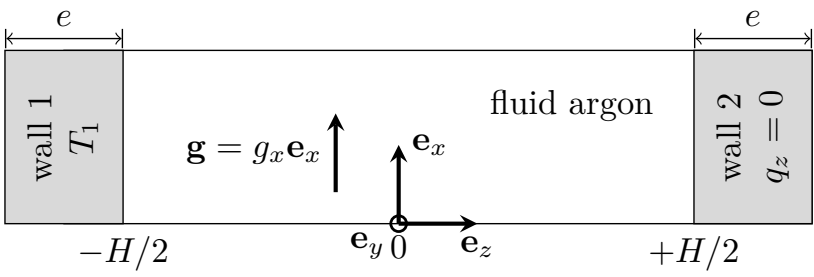

Figure 1: Physical configuration

For the compressible fluid flow in the micro-channel of length $L$, driven by the pressure difference $p_{\text {in }}-p_{\text {out }}$, the density decreases all along the channel. From the mass flow rate conservation, the velocity magnitude increases, and consequently, the viscous heating also. If the length scale associated with the pressure gradient is assumed much larger that the channel height, namely $\left(p_{\text {in }}+p_{\text {out }}\right) /\left(p_{\text {in }}-p_{\text {out }}\right) \gg 2 H / L$, then the density variations over a length scale $H$ of the channel can be disregarded. Therefore the axial dependence of all the macroscopic variables are locally negligible, at least far from the inlet and outlet regions [14]. Thus, in what follows, the thermodynamics quantities are assumed to depend only on the transverse coordinate $z$.

From a macroscopic point-of-view, and as discussed in [15], the total energy flux $\mathbf{q}_{t o t}^{(c)}$ in the fluid region can be split into three contributions:

$$
\begin{aligned}
\mathbf{q}_{t o t}^{(c)} & =\mathbf{q}_{k}+\mathbf{q}_{\mu}+\mathbf{q}_{h} \\
& =-k_{f} \nabla T-(\overline{\bar{\tau}} \cdot \mathbf{u})+\rho h \mathbf{u}
\end{aligned}
$$

where $\mathbf{q}_{k}=-k_{f} \nabla T$ is the conduction heat flux with $k_{f}$ the fluid conductivity, $\mathbf{q}_{\mu}=-(\overline{\bar{\tau}} \cdot \mathbf{u})$ is the energy flux produced by the viscous friction and $\mathbf{q}_{h}=\rho h \mathbf{u}$ is the convection enthalpy flux. Since the velocity simply writes $\mathbf{u}=u_{x}(z) \mathbf{e}_{x}$, the total energy flux in $z$-direction, $q_{\text {tot }}^{(c)}$, reduces into two terms in $z$-direction:

$$
q_{\text {tot }}^{(c)}=-k_{f} \frac{\partial T}{\partial z}-u_{x} \mu \frac{\partial u_{x}}{\partial z}
$$

The Maslen's flux boundary condition [10] is to equalize the total energy fluxes on both sides of the fluid/solid interface. In the wall region, the total energy flux reduces in its conduction contribution:

$$
-\left.k_{s} \frac{\partial T}{\partial z}\right|_{H / 2}=-\left.k_{f} \frac{\partial T}{\partial z}\right|_{H / 2}-\left.u_{x} \mu \frac{\partial u_{x}}{\partial z}\right|_{H / 2}
$$

where $k_{s}$ is the solid conductivity and $\mu$ the fluid dynamic viscosity. In order to check the validity of Eq. (3) in the Knudsen layer, simulations are carried out at the molecular scale.

\section{Microscopic approach}

Solid walls are composed of platinum atoms disposed on a FCC111 lattice. The dynamics of all atoms, either argon confined between the solid walls or platinum, relies on the classical Newton's law. The trajectory of each atom $i$, or particle, is governed by $\mathbf{F}_{i}=m_{i} \ddot{\mathbf{r}}_{i}$ where $\ddot{\mathbf{r}}_{i}$ is the acceleration vector, $m_{i}$ is the mass and $\mathbf{F}_{i}$ is the resultant of external forces acting on particle $i$. With $\mathbf{r}_{i}$ is the particle position, $\mathbf{g}$ is the volume force which drives the fluid flow, and assuming only binary interactions given by the pairwise potential $U_{\alpha / \beta}$, the resultant $\mathbf{F}_{i}$ is developed as $\mathbf{F}_{i}=-\nabla\left[\mathbf{g} \cdot\left(\mathbf{r}_{i}-\left\langle\mathbf{r}_{i}\right\rangle\right)+\sum_{j} U_{\alpha / \beta}\left(r_{i j}\right)\right]$ for an argon particle and is reduced to $\mathbf{F}_{i}=-\nabla \sum_{j} U_{\alpha / \beta}\left(r_{i j}\right)$ for a platinum one. The classical Lennard-Jones model [16] is chosen to describe the argon/argon, platinum/platinum and argon/platinum interactions:

$$
U_{\alpha / \beta}\left(r_{i j}\right)=4 \varepsilon_{\alpha / \beta}\left[\left(\frac{\sigma_{\alpha / \beta}}{r_{i j}}\right)^{12}-\left(\frac{\sigma_{\alpha / \beta}}{r_{i j}}\right)^{6}\right]
$$

where $\alpha$ and $\beta$ denote the Ar- or Pt-atoms, $r_{i j}=\left\|\mathbf{r}_{i j}\right\|$ is the intermolecular distance between the $i$ and $j$ particles, $\mathbf{r}_{i j}=\mathbf{r}_{j}-\mathbf{r}_{i}, \varepsilon_{\alpha / \beta}$ is the potential welldepth and $\sigma_{\alpha / \beta}$ is the distance where the potential cancels out. Taking argon as reference for the physical properties, the interaction coefficients write $\varepsilon_{A r / A r}=$ $1 \varepsilon, \sigma_{A r / A r}=1 \sigma, m_{A r / A r}=m, \varepsilon_{P t / P t}=31.3 \varepsilon$, $\sigma_{P t / P t}=0.73 \sigma, \varepsilon_{A r / P t}=0.66 \varepsilon, \sigma_{A r / P t}=0.865 \sigma$, $m_{P t}=4.8833 m$ with $\varepsilon=1.656 \times 10^{-21} \mathrm{~J}, \sigma=3.405 \AA$ and $m=6.633 \times 10^{-26} \mathrm{~kg}$. The reference scale expressions and their derived quantities are given in Table 1.

\begin{tabular}{rrrl}
\hline Scale & Expression & Value & \\
\hline \hline mass (Ar) & $m$ & $6.633 \times 10^{-26}$ & $\mathrm{~kg}$ \\
length & $\sigma$ & $3.405 \times 10^{-10}$ & $\mathrm{~m}$ \\
energy & $\varepsilon$ & $1.656 \times 10^{-21}$ & $\mathrm{~J}$ \\
\hline \hline time, $t_{0}$ & $\sqrt{m \sigma^{2} / \varepsilon}$ & $2.155 \times 10^{-12}$ & $\mathrm{~s}$ \\
temperature, $T_{0}$ & $\varepsilon / k_{B}$ & 120 & $\mathrm{~K}$ \\
velocity, $v_{0}$ & $\sqrt{\varepsilon / m}$ & 158.03 & $\mathrm{~m} / \mathrm{s}$ \\
density, $\rho_{0}$ & $m / \sigma^{3}$ & 1680.31 & $\mathrm{~kg} / \mathrm{m}^{3}$ \\
force, $g_{0}$ & $\varepsilon / \sigma$ & $4.86 \times 10^{-12}$ & $\mathrm{~N}$ \\
viscosity, $\mu_{0}$ & $\sqrt{m \varepsilon} / \sigma^{2}$ & $9.0345 \times 10^{-5}$ & $\mathrm{~kg} / \mathrm{m} / \mathrm{s}$ \\
conductivity, $k_{0}$ & $k_{B} \sqrt{\varepsilon / m} / \sigma^{2}$ & 0.0188 & $\mathrm{~W} / \mathrm{m} / \mathrm{K}$ \\
heat flux, $q_{0}$ & $\sqrt{\varepsilon^{3} / m} / \sigma^{3}$ & $6.62 \times 10^{9}$ & $\mathrm{~W} / \mathrm{m}^{2}$ \\
\hline
\end{tabular}

Table 1: Physical scales, expressions and values used in the molecular dynamics simulations, with $k_{B}$ is the Boltzmann constant

The choice of argon as a fluid is justified by the binary interactions and spherical approximation induced by the pairwise Lennard-Jones potential choice. This latter allows a good restitution of the dynamical and 
thermal properties of mono-atomic gases, and to some extend, of non-spherical molecules for dense cases [1, $6]$.

Like in continuum mechanics (Sec. 2), the total energy flux $\mathbf{q}_{\text {tot }}^{(m)}$ can be defined from a microscopic description. Using the Irving-Kirkwood formulation [3, 16], it reads:

$$
\begin{aligned}
\mathbf{q}_{t o t}^{(m)}= & \frac{1}{2 V} \sum_{i}^{N} m_{i} \dot{\mathbf{r}}_{i}^{2} \dot{\mathbf{r}}_{i} \\
& +\frac{1}{2 V} \sum_{i}^{N} \sum_{j \neq i}^{N} U_{\alpha / \beta}\left(r_{i j}\right) \dot{\mathbf{r}}_{i} \\
& -\frac{1}{2 V} \sum_{i}^{N} \sum_{j \neq i}^{N} \mathbf{r}_{i j}\left[\mathbf{f}_{i j} \cdot \dot{\mathbf{r}}_{i}\right]
\end{aligned}
$$

where $\dot{\mathbf{r}}_{i}$ is the velocity of particle $i, \mathbf{f}_{i j}=-\nabla U_{\alpha / \beta}\left(r_{i j}\right)$ is the interaction force between particles $i$ and $j$ and $N$ is the total number of particles in the volume $V$ where $\mathbf{q}_{t o t}^{(m)}$ is evaluated. The first and second terms of the r.h.s. are related to summations of kinetic and potential energy carried by a particle $i$, while the third term represents the energy transfer by the pressure work [9]. It can be noticed that no contribution involving the volume force $\mathbf{g}$ exists, simply because the velocity and position are independent variables.

In order to impose a zero conduction heat flux in the solid platinum wall ranging from $z=H / 2$ to $z=H / 2+e$, the fluid region is replicated at the wall ending, at $z=H / 2+e$. That trick also allows keeping the periodicity of molecular simulations in all space directions. Since the potential interaction between two particles decreases quickly with their distance, to become negligible in the total amount of energy, the computation cost is highly reduced by introducing a truncation length $r_{c}$. In practice, the interaction force derived from Eq. (4) is set to zero, if $r_{i j}>r_{c}$. The truncation radius $r_{c}$ is set to $r_{c}=2.5 \sigma$.

The simulations are carried out on boxes of dimensions $\ell_{x} \times \ell_{y} \times \ell_{z}$. With the fluid zone replica, the length is finally $\ell_{z}=2(H+e)$. The lengths $\ell_{i}, i=x, y$, are multiples of the gaps of the FCC111 solid lattice. They also fulfil the condition $\ell_{i} \geq 3 \times r_{c}$ in order to manage efficiently the neighbourhood of each particle (Cell and Verlet lists [16]). The height $H$ is such that $H \geq C \times \min \left(\ell_{x}, \ell_{z}\right)$ and is adjusted to fit the desired fluid density $\rho$ in the volume defined by $V=\ell_{x} \times \ell_{y} \times$ $H$. The constant $C$, initially fixed at $C=10$, can be adapted to change the $K n$ number. In order the particles in both fluid regions do not interact between each other, the wall thickness $e$ is such that $e \geq r_{c}$ and $e$ is a multiple of the vertical gap of the solid FCC111 lattice.

The temperature of the left wall is maintained at $T_{1}$ using a Langevin thermostat [7], while that of the opposite wall is left free. The Velocity-Verlet algorithm [16] is used to perform the time integration with a time step fixed at $\Delta t / t_{0}=5 \times 10^{-3}$.
To calculate the macroscopic quantities $\rho, \mathbf{u}, T$ and $\mathbf{q}_{t o t}^{(m)}$, time and space averages are performed on $n_{z}$ uniform sub-domains covering the channel height $H$ and over time intervals $\tau_{a}$. The converged estimate of the variance of the ensemble average is computed by a block average technique $[4,5]$.

\section{Results and discussions}

The molecular dynamics simulations are based on a Lennard-Jones potential interactions. Therefore, the pressure, the temperature and the density are solutions of a specific equation of state [8]. Likewise, the dynamic viscosity $\mu$ and the thermal conductivity $k_{f}$ are estimated through the correlations by Galliéro et al. [6], and Bugel and Galliéro [1]. The results are then presented as a function of the physical quantities, namely the mean density $\rho$ and the volume force $g_{x}$. The magnitude order of the slip velocity and jump temperature is controlled by the Knudsen number

$$
K n=\frac{\lambda}{2 H}
$$

with $\lambda$ is the mean free path for molecules interacting through long-range potentials, which is expressed with the formulation by Cercignani [2]:

$$
\lambda=\frac{\mu}{\rho} \sqrt{\frac{\pi m}{2 k_{B} T}}
$$

where $m$ is the molecular mass, $T$ is the temperature of the gas, and $k_{B}$ is the Boltzmann constant.

For the sake of simplicity, the physical parameters and the dynamic and thermal variables, averaged over the channel height, are gathered in Table 2 as a function of

\begin{tabular}{rrllll}
\hline$\rho / \rho_{0}$ & $g_{x} / g_{0}$ & $H / \sigma$ & $\bar{u}_{x} / v_{0}$ & $\bar{\theta}_{b} / T_{0}$ & $\left|\bar{q}_{\text {tot }}^{(m)}\right| / q_{0}$ \\
\hline \hline 0.6 & 0.02 & 19.87 & 0.882 & 1.61 & 0.105 \\
0.6 & 0.01 & 19.87 & 0.452 & 0.534 & 0.027 \\
0.6 & 0.005 & 19.87 & 0.225 & 0.152 & 0.0067 \\
0.6 & 0.0025 & 19.87 & 0.112 & 0.042 & 0.0011 \\
0.6 & 0.002 & 96.65 & 1.171 & 3.135 & 0.066 \\
0.1 & 0.01 & 28.05 & 0.719 & 2.59 & 0.0097 \\
0.05 & 0.005 & 51.28 & 0.713 & 2.42 & 0.0045 \\
\hline
\end{tabular}

Table 2: Normalization parameters for Figs. 3-8. The Knudsen number is $K n=0.036$, except the simulation with $g_{x} / g_{0}=0.002$ where $K n=0.0075$

the Knudsen number $K n$, the mean fluid density $\rho$ and the volume force $g_{x}$. The notation $\bar{\bullet}$ denotes a spatial average over the channel height. All molecular dynamics simulations were carried out for a thermostated wall fixed at $T_{1} / T_{0}=1.5$. The mean free path (Eq. 7 ), and therefore the Knudsen number (Eq. 6), is evaluated for a fluid flow without volume force $g_{x}=0\left(u_{x}=0\right)$, at the temperature equilibrium $\left(T=T_{1}\right)$ and with a density measured in the fluid core, far from the boundaries. 


\subsection{Total energy flux analysis}

The first simulation is carried out for a fluid density $\rho / \rho_{0}=0.6$ and a volume force $g_{x} / g_{0}=0.01$. Based on the expression of the mean free path (Eq. 7), the Knudsen number (6) is $K n=0.036$. According to the fluid flow regimes, a velocity slip and a temperature jump are expected at the fluid/solid interface.

In order to compare the total energy flux at the molecular scale $q_{\text {tot }}^{(\mathrm{m})}$ (Eq. 5) with its continuous counterpart $q_{\mathrm{tot}}^{(\mathrm{c})}$ (Eq. 2), the conduction heat flux $q_{k}$ and the viscous power density $q_{\mu}$ have to be evaluated. To this end, the macroscopic velocity and temperature are first calculated by averaging the particle velocities and the local kinetic energy fluctuations (see Fig. 5 and Fig. 6 for the velocity and temperature profiles). Then, the macroscopic fluxes are approximated using the second order centred finite difference scheme to get the $z$-derivatives, combined with the dynamic viscosity and thermal conductivity provided by correlations $[1$, 6]. In Fig. 2 are presented the different fluxes $q_{\mathrm{tot}}^{(\mathrm{m})}$,

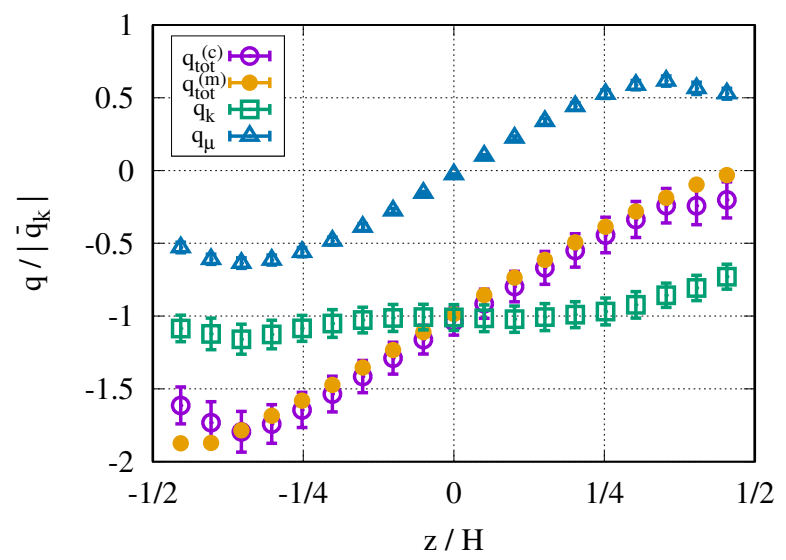

Figure 2: Normalized fluxes with respect to $\left|\bar{q}_{k}\right| / q_{0}=$ $2.74 \times 10^{-2}$, the $z$-average of the conduction heat flux, for $K n=0.036, \rho / \rho_{0}=0.6$ and $g_{x} / g_{0}=0.01$

$q_{\mathrm{tot}}^{(\mathrm{c})}, q_{k}$ and $q_{\mu}$ the viscous contribution to the energy equation. Each of them is normalized with respect to the absolute value of the mean conduction heat flux over the channel height. The error bars take into account the uncertainties on both, the calculated macroscopic data, the conductivity and viscosity correlations and the approximations of the velocity and temperature derivatives as well. The analysis of the curves confirms that the total energy flux computed with the molecular dynamics, $q_{t o t}^{(m)}$, gets to zero at the fluid/solid interface $z / H=1 / 2$, which is consistent with the conduction heat flux transmitted to the adiabatic solid wall. The reconstructed macroscopic flux $q_{t o t}^{(c)}$ exhibits a very good agreement with the molecular one $q_{t o t}^{(m)}$ in the core channel, and to a lesser extent, near the fluid/solid interfaces. In this problem, the viscous contribution $q_{\mu}$ is far from being negligible, since it reaches almost half of the conduction heat flux. Its spatial distribution is anti-symmetric with respect to $z=0$, which results from the symmetry of the nearly parabolic velocity profile (Fig. 5). Because the temperature profile is almost linear (Fig. 6), the conduction heat flux $q_{k}$ is nearly constant; the variations are mainly due to the conductivity dependence on the density and temperature. Finally, the macroscopic energy flux decomposition (2) is, as expected, accurate in the fluid core domain. On the other hand, differences are observed in the vicinity of the walls. At the adiabatic fluid/solid interface, the reconstructed flux is not as small as the molecular one, what may suggest that the macroscopic expression (2) fails to represent the right total energy flux, at least locally.

In order to examine thoroughly the departures between the total energy fluxes $q_{\text {tot }}^{(m)}$ and $q_{\text {tot }}^{(c)}$, calculated by either the Irving-Kirkwood formulation (Eq. 5) or deduced from relations of the continuum mechanics (Eq. 2), their relative difference to $\bar{q}_{t o t}^{(m)}$ is drawn for different densities, external forces and Knudsen numbers (Fig. 3). The reconstructed fluxes $q_{\text {tot }}^{(c)}$ deviate

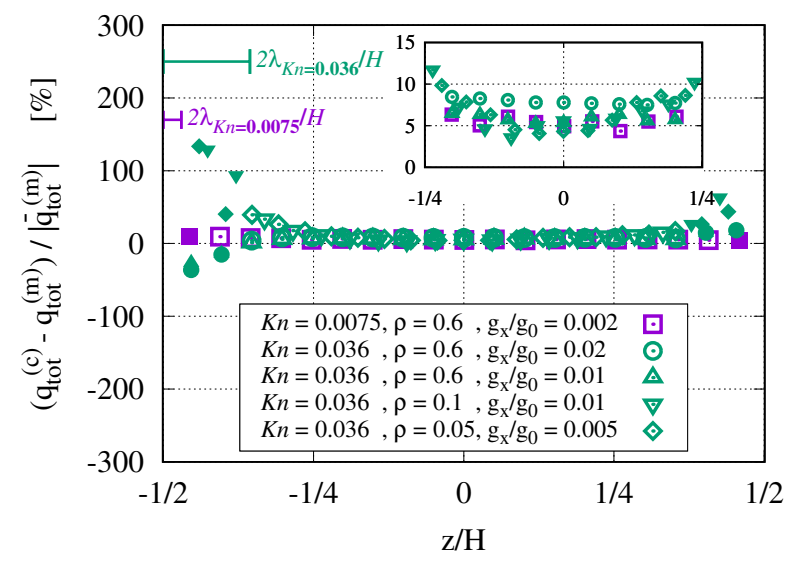

Figure 3: Relative difference between the total energy fluxes $q_{\text {tot }}^{(m)}$ (Eq. 5) and $q_{t o t}^{(c)}$ (Eq. 2) reconstructed with the molecular data. Filled (empty) symbols stand for points inside (outside) the Knudsen layer $(|z| / H-1 / 2)<2 \lambda / H=4 K n$. For clarity reason, the error bars have been omitted. Reference values are given in Table 2

by less than $10 \%$ from the molecular ones $q_{t o t}^{(m)}$ in the bulk fluid domain. As discussed before, the discrepancies are mainly due to the reconstructed flux $q_{t o t}^{(c)}$ which relies on, both correlation laws for the thermal conductivity and dynamic viscosity evaluations, and approximations of the derivatives by the second order centred difference scheme. However, in a thickness region of about two mean free paths, the relative gap increases substantially, all the more that one gets closer to the wall. Looking at the density distributions (Fig. 4), growing oscillations develop towards the solid wall, approximately over the same length scale. These oscillations result from the clustering of the argon atoms close to the wall. Since the physical properties are density dependent, the dynamic viscosity $\mu$ and the thermal conductivity $k_{f}$ fluctuate in this zone as well. 
This atomic patterning breaks the local thermodynamics equilibrium, which is required to get a macroscopic model for the fluid flow. The aim of the slip/jump relations is precisely to connect the macroscopic temperature and velocity variables on both side of the Knudsen layer, namely between the wall and the outer region of the Knudsen layer. The use of these boundary conditions, and more specifically the Maslen's flux condition, is the motivation of what follows.

\subsection{Comparison between the molecular and continuum models}

This section is devoted to the comparisons of the molecular dynamics results with those derived from the continuum model with constant physical properties, an incompressible fluid flow assumption and suitable boundary conditions. The simulations are carried out for volume force ranging from $g_{x} / g_{0}=0.0025$ to 0.02 and a mean density $\rho / \rho_{0}=0.6$. The Knudsen number, evaluated at $T_{1}$, is equal to $K n=0.036$.

The assumption of a constant density is acceptable in the core flow, where a weak decrease is observed due to the heating by the viscous friction (Fig. 4). On the

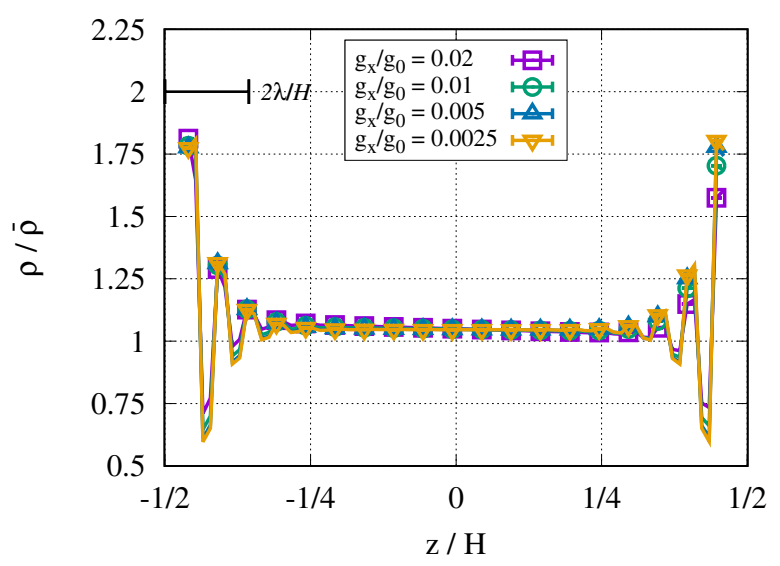

Figure 4: Density $\rho(z) / \bar{\rho}$ for different volume forces $g_{x} / g_{0}$ and $K n=0.036$ (see Table 2). The number of bins for the molecular average calculation is $n_{z}=100$

other hand, at the vicinity of the boundaries, the density oscillates and departs up to about $75 \%$ of its bulk value. As already mentioned, this usual behaviour is related to the clustering of the argon atoms near solid wall due to the interaction parameters that make the Lennard-Jones potential attractive [13]. The oscillations are damped over a length of approximately $2 \lambda$ that roughly corresponds to the Knudsen layer.

The normalized velocity profiles, presented in Fig. 5, exhibit a Poiseuille like flow with slip at walls. Whereas the magnitude of the velocity and the dynamical slip depend on the applied volume force $g_{x}$, they become quite identical once they are normalized by $\bar{u}_{x}$, the averaged value of $u_{x}$ over the channel height (Table 2). The superposition of the molecular data are fully consistent with the analytical function derived from the

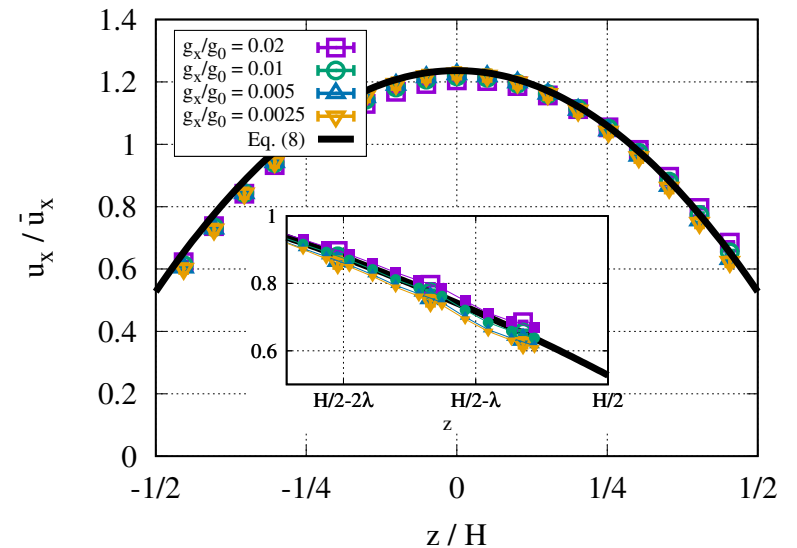

Figure 5: Velocity $u_{x}(z) / \bar{u}_{x}$ for different volume forces $g_{x} / g_{0}$ and $K n=0.036$. The average values are provided in Table 2. The parameters for the analytical function is $\sigma_{\mu}=2.46$ and $\xi=36.0$. The number of bins for the molecular average calculation is $n_{z}=25$, except the encapsulated figures which are based on $n_{z}=100$

continuum model:

$$
\frac{u_{x}(z)}{\bar{u}_{x}}=\frac{3\left(1+8 \sigma_{\mu} K n\right)-12(z / H)^{2}}{2+24 \sigma_{\mu} K n}
$$

with $\sigma_{\mu}$ is the viscous slip coefficient classically used in the expression of the dynamical slip boundary condition:

$$
u_{f}-u_{w}=\left.\sigma_{\mu} \lambda\left(\nabla u \cdot \mathbf{n}_{w f}\right)\right|_{f}
$$

The indexes $f$ and $w$ indicate fluid and wall quantities evaluated at the fluid/solid interface and $\mathbf{n}_{w f}$ its unit normal vector, directed from the wall to the fluid. In the present problem, $u_{w}=0$.

Since the velocity profile is not constant in the channel section, the viscous friction leads to mechanical energy dissipation and a temperature increase by getting away from the lower wall at $T_{1}$. This heating and the temperature jump are even more pronounced as the external force $g_{x}$ increases. However, like for the velocity, the dimensionless reduced temperature $\theta / \theta_{b}$, with $\theta=T-T_{1}$ and $\theta_{b}=\int \rho u_{x} \theta d z / \int \rho u_{x} d z$ the dimensionless bulk temperature (Table 2), turns out to be independent on the external force $g_{x}$ (Fig. 6). Such a result is recovered by integrating the energy equation with the velocity field (8), the temperature jump at the lower boundary $z / H=-1 / 2$

$$
T_{f}-T_{w}=\left.\xi \lambda\left(\nabla T \cdot \mathbf{n}_{w f}\right)\right|_{f}
$$

with $\xi$ the thermal jump coefficient, and the Maslen's flux condition (3) at the adiabatic wall $z / H=$ $1 / 2$. The analytical reduced temperature $\theta(z) / \theta_{b}$ then reads:

$$
\frac{\theta(z)}{\theta_{b}}=A+B \frac{z}{H}+C\left(\frac{z}{H}\right)^{4}
$$

with

$$
\begin{aligned}
& A=\frac{a_{0}+a_{1} K n+a_{2} K n^{2}}{D}, B=\frac{96 \sigma_{\mu} K n+8}{D}, C=\frac{-16}{D}, \\
& D=\frac{2304\left(d_{0}+d_{1} K n+d_{2} K n^{2}+d_{3} K n^{3}\right)}{\left(12 \sigma_{\mu} K n+1\right)}
\end{aligned}
$$




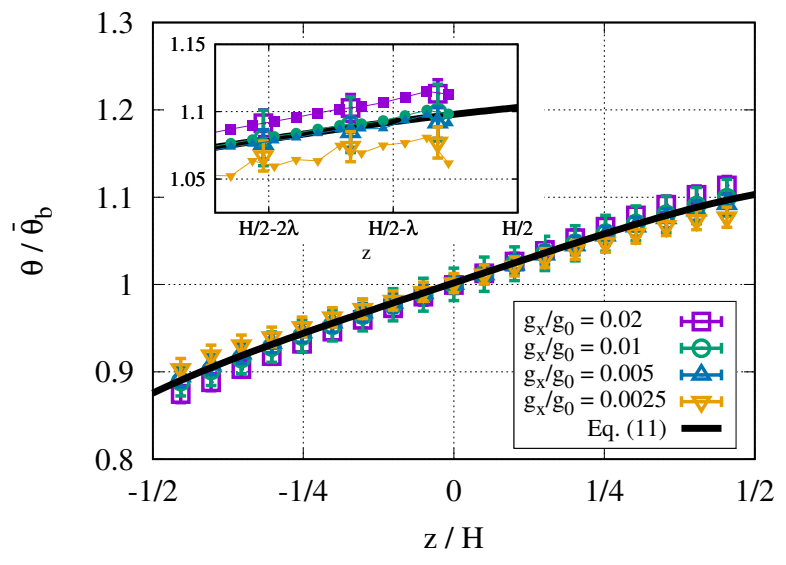

Figure 6: Same caption as Fig. 5 - Reduced temperature $\theta(z) / \theta_{b}$

and

$$
\begin{aligned}
& a_{0}=5, a_{1}=48 \sigma_{\mu}+32 \xi, a_{2}=192 \sigma_{\mu} \xi \\
& d_{0}=\frac{43}{20160}, d_{1}=\frac{\xi}{72}+\frac{11 \sigma_{\mu}}{240} \\
& d_{2}=\frac{\sigma_{\mu}}{4}\left(\xi+\sigma_{\mu}\right), d_{3}=\sigma_{\mu}^{2} \xi
\end{aligned}
$$

This analytical solution, which is based on the Maslen's flux condition, fits perfectly the molecular data. This observation suggests that the flux boundary condition (3), which can be interpreted as a jump in the conduction heat flux, permits to connect correctly the thermal quantities on both part of the Knudsen layer. As well as the velocity and temperature profiles, the total energy flux becomes independent of the volume force $g_{x}$, once the suitable normalization $q_{t o t}^{(m)} /\left|\bar{q}_{t o t}^{(m)}\right|$ is considered (Fig. 7). An analytical approximation

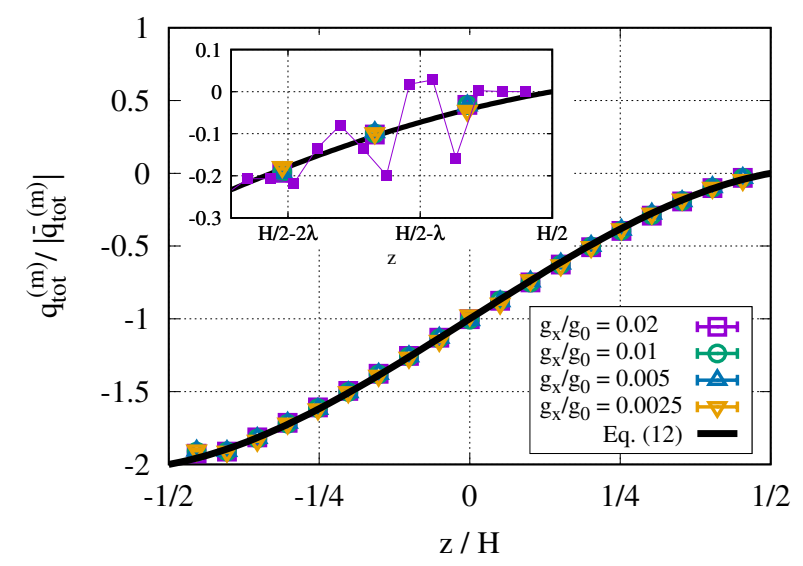

Figure 7: Same caption as Fig. 5 - Total energy flux $q_{\text {tot }}^{(m)}(z) /\left|\bar{q}_{\text {tot }}^{(m)}\right|$.

$q_{\text {tot }}^{(a)}(z)$ of the molecular data is developed by substituting the velocity (8) and temperature (11) functions into the macroscopic expression of the total energy flux (2):

$$
\frac{q_{\text {tot }}^{(a)}(z)}{\left|\bar{q}_{\text {tot }}^{(a)}\right|}=\frac{\left(24 \sigma_{\mu} K n+3\right)(z / H)-4(z / H)^{3}}{12 \sigma_{\mu} K n+1}-1
$$

Again, the analytical expression provides a very good approximation of the results coming from the molecular dynamics simulations. It is worth to noticed that, as for the density profiles (Fig. 4), flux oscillations are observed at the molecular scale, over a length of about $2 \lambda$.

To point out the robustness of the Maslen's boundary conditions, the total heat fluxes $q_{t o t}^{(m)}$, achieved by the molecular simulations and presented in Fig. 3, are reconsidered and compared to the analytical solutions $q_{\text {tot }}^{(a)}(z)$ (Fig. 8). Taking into account the

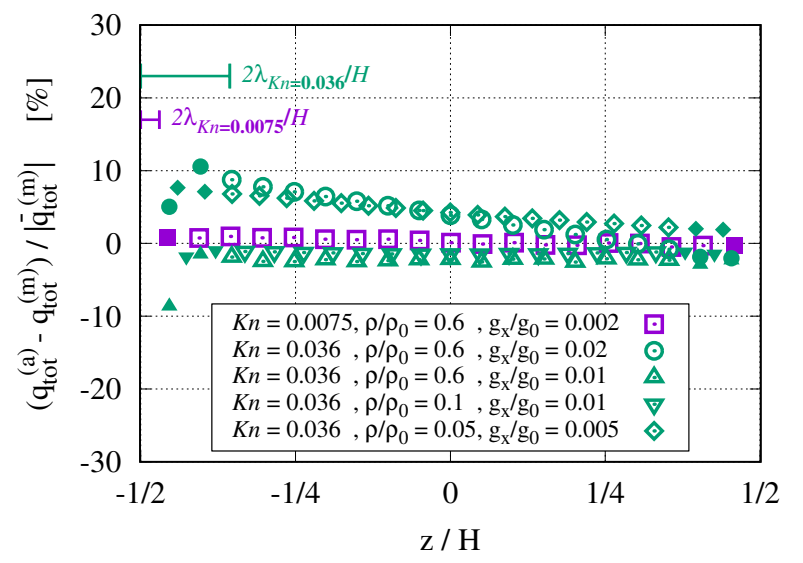

Figure 8: Relative gap between the total energy fluxes stemmed from molecular dynamics simulations and the analytical expression (12). Filled symbols stand for points inside the Knudsen layer $(|z| / H-1 / 2)<2 \lambda$, while empty ones denote measurement points in the bulk. For clarity reason, the error bars have been omitted. Reference values are given in Table 2

approximation level used to develop the continuum model, the analytical function $q_{t o t}^{(a)}(z)$ gives satisfying results, with relative gaps less than $10 \%$. The accuracy is much better close to the adiabatic wall $z / H=1 / 2$ $(<2 \%)$, where the total energy flux is controlled by the Maslen's condition. The slight deviation observed by moving away from the adiabatic wall is probably the result of error accumulations induced by the assumptions used to develop the analytical solution. Again, the comparisons between the analytical and molecular total energy fluxes emphasize the robustness of the Maslen's flux boundary condition (3) to achieve a correct approximation.

From the dimensionless writing of the Navier-Stokes and energy equations with their boundary conditions, 6 dimensionless parameters appear: the heat conductivity ratio $k_{s} / k_{f}$, the ratio between the volume and viscous forces measured by the Jeffreys number $J e=g_{x} H^{2} /(\mu U)$, the energy flux ratio between the viscous friction and the heat conduction, namely the Brinkman number $B r=\mu U^{2} /\left(k_{f} \Delta T\right)$, and obviously the above introduced Knudsen number $K n$ and the slip and jump coefficients $\sigma_{\mu}$ and $\xi$. Setting $J e=1$ leads to define the scale for the velocity: 
$U=g_{x} H^{2} / \mu$. Likewise, the equilibrium between the viscous dissipation and the heat conduction allows to define the scale for the temperature variation: $\Delta T=\mu U^{2} / k_{f}=g_{x}^{2} H^{4} / \mu k_{f}$. Table 3 summarizes

\begin{tabular}{rrrrrr}
\hline$\rho / \rho_{0}$ & $K n$ & $J e$ & $B r$ & $\sigma_{\mu}$ & $\xi$ \\
\hline \hline 0.6 & 0.0075 & 9.6 & 0.13 & 2.63 & 36 \\
0.6 & 0.036 & 5.1 & 0.24 & 2.46 & 36 \\
0.1 & 0.036 & 5.5 & 0.21 & 4.13 & 37 \\
0.05 & 0.036 & 5.2 & 0.15 & 5.72 & 25 \\
\hline
\end{tabular}

Table 3: Dimensionless parameters for simulations presented in Figs. 3 and $8 ; \sigma_{\mu}$ and $\xi$ are used in the analytical functions (8), (11) and (12).

the Jeffreys, Brinkman and Knudsen parameters, and the slip and jump coefficients as well. For each fixed Knudsen, the numerical values $\sigma_{\mu}$ and $\xi$ result from a minimization process between the equations (8), (11) and (12) and the averaged molecular data, using the non-linear least-squares Marquardt-Levenberg algorithm. Since the fluid properties results from the Lennard-Jones interaction potential, the ranges of variation of the the Jeffreys and Brinkman parameters are moderate: the ratio between the maximal and minimal values are close to 2 .

The numerical parameters involved in the molecular dynamics simulations, such as the cut-off radius $r_{c}$, the time step $\Delta t$, the lengths $\ell_{x}$ and $\ell_{y}$, the interaction potential of the platinum atoms of the solid wall or the $(\sigma, \varepsilon)$-values of the $\mathrm{Ar} / \mathrm{Pt}$ interaction coefficients do not alter the analyses presented below. Obviously, the changes in the Lennard-Jones interaction parameters may modify slightly the fluid flow and heat transfer, especially the dynamical slip and temperature jump magnitudes, but the velocity, temperature, and flux profiles remain similar; the total energy fluxes computed by the molecular simulation and the analytical model, using the Maslen's flux boundary condition (3), always cancel at the adiabatic solid wall $z=H / 2$.

\section{Conclusion}

Results of molecular dynamics simulation, obtained for a Lennard-Jones fluid, have shown that the Maslen's boundary condition [10], demonstrated in the framework of the continuum mechanics (see [15]), although questionable in the Knudsen layer, is proved to be efficient to connect the conduction heat fluxes between the wall and the fluid domain. Therefore, the total energy flux conservation at the fluid/solid interface introduces effectively a jump in the conduction heat flux which is equal to the energy flux produced by the viscous friction.

\section{References}

[1] Bugel, M. and Galliéro, G. "Thermal conductivity of the Lennard-Jones fluid: An empirical correlation". In: Chemical Physics 352.1 (2008), pp. 249-257.

[2] Cercignani, C. Slow rarefied flows: Theory and application to Micro-Electro-Mechanical Systems. Vol. 41. Springer Science \& Business Media, 2006.

[3] Fan, Z., Pereira, L. F. C., Wang, H.-Q., Zheng, J.-C., Donadio, D., and Harju, A. "Force and heat current formulas for many-body potentials in molecular dynamics simulations with applications to thermal conductivity calculations". In: Phys. Rev. B 92 (9 2015), p. 094301.

[4] Flyvbjerg, H. and Petersen, H. G. "Error estimates on averages of correlated data". In: The Journal of Chemical Physics 91.1 (1989), pp. 461-466.

[5] Frenkel, D. and Smit, B. Understanding Molecular Simulation. Second Edition. San Diego: Academic Press, 2002, pp. 525-532.

[6] Galliéro, G., Boned, C., and Baylaucq, A. "Molecular Dynamics Study of the LennardJones Fluid Viscosity: Application to Real Fluids". In: Industrial \& Engineering Chemistry Research 44.17 (2005), pp. 6963-6972.

[7] Hünenberger, P. H. "Thermostat Algorithms for Molecular Dynamics Simulations". In: Advanced Computer Simulation: Approaches for Soft Matter Sciences I. Ed. by C. Dr. Holm and K. Prof. Dr. Kremer. Berlin, Heidelberg: Springer Berlin Heidelberg, 2005, pp. 105-149.

[8] Kolafa, J. and Nezbeda, I. "The Lennard-Jones fluid: An accurate analytic and theoreticallybased equation of state". In: Fluid Phase Equilibria 100 (1994), pp. 1-34.

[9] Maruyama, S. "Molecular dynamics methods in microscale heat transfer". In: Advances in $\mathrm{Nu}$ merical Heat Transfer 2.6 (2002), 189-226.

[10] Maslen, S. H. "On Heat Transfer in Slip Flow". In: Journal of the Aerospace Sciences 25.6 (1958), pp. 400-401.

[11] Maxwell, J. C. "VII. On stresses in rarified gases arising from inequalities of temperature". In: Philosophical Transactions of the Royal Society of London 170 (1879), pp. 231-256.

[12] Navier, C. "Mémoires de l'Académie Royale des Sciences de l'Institut de France". In: vol. 6. 1823. Chap. Mémoire sur les lois du mouvement des fluides, pp. 389-440.

[13] Nedea, S., Steenhoven, A. van, Markvoort, A., Spijker, P, and Giordano, D. "Gas-surface interactions using accommodation coefficients for a dilute and a dense gas in a micro-or nanochannel: Heat flux predictions using combined molecular dynamics and Monte Carlo techniques". In: Physical Review E 89.5 (2014), p. 053012. 
[14] Nicolas, X., Chénier, E., Tchekiken, C., and Lauriat, G. "Revisited analysis of gas convection and heat transfer in micro channels: Influence of viscous stress power at wall on Nusselt number". In: International Journal of Thermal Sciences 134 (2018), pp. 565-584.

[15] Nicolas, X., Chénier, E., and Lauriat, G. "Thermal boundary conditions for convective heat transfer of dilute gases in slip flow regime". In: International Journal of Thermal Sciences 135 (2019), pp. 298-301.

[16] Rapaport, D. C. The Art of Molecular Dynamics Simulation. 2nd ed. Cambridge University Press, 2004. 